\title{
FAULT DETECTION AND LOCALIZATION IN MOTORCYCLES BASED ON THE CHAIN CODE OF PSEUDOSPECTRA AND ACOUSTIC SIGNALS
}

\author{
B. S. Anami ${ }^{1}$ and V. B. Pagi ${ }^{2}$ \\ ${ }^{1}$ KLE Institute of Technology, \\ Opp. Airport, Gokul, Hubli - 580 030, India \\ ${ }^{2}$ Faculty of Computer Applications, Basaveshwar Engineering College \\ S. Nijalingappa Vidyanagar, Bagalkot - 587102, India \\ Phone: +919342647100; Fax: +918354234204 \\ Email: veereshpagi@yahoo.com
}

\begin{abstract}
Vehicles produce sound signals with varying temporal and spectral properties under different working conditions. These sounds are indicative of the condition of the engine. Fault diagnosis is a significantly difficult task in geographically remote places where expertise is scarce. Automated fault diagnosis can assist riders to assess the health condition of their vehicles. This paper presents a method for fault detection and location in motorcycles based on the chain code of the pseudospectra and Mel-frequency cepstral coefficient (MFCC) features of acoustic signals. The work comprises two stages: fault detection and fault location. The fault detection stage uses the chain code of the pseudospectrum as a feature vector. If the motorcycle is identified as faulty, the MFCCs of the same sample are computed and used as features for fault location. Both stages employ dynamic time warping for the classification of faults. Five types of faults in motorcycles are considered in this work. Observed classification rates are over $90 \%$ for the fault detection stage and over $94 \%$ for the fault location stage. The work identifies other interesting applications in the development of acoustic fingerprints for fault diagnosis of machinery, tuning of musical instruments, medical diagnosis, etc.
\end{abstract}

Keywords: Motorcycle fault diagnosis; pseudospectral analysis; acoustic signal; DTW classifier.

\section{INTRODUCTION}

Motorcycles are the favorite mode of transport in India. They account for the majority of the Indian automobile market with nearly $77 \%$ of the share of total vehicle sales. Two-wheelers registered a growth of $15.94 \%$ during April-August 2011. Unit sales of motorcycles are estimated to exceed 10 million by 2012-13 (IR, 2010). Vehicles produce different sound patterns under different working conditions when in motion. Service mechanics can diagnose faults based on the sounds produced by using the expertise acquired over many years. Vehicle classification and fault diagnosis based on acoustic signals adds further difficulty to analyzing sound patterns. The most influential factors include the non-stationary nature of the sound, and variations in speed, engine condition, road condition, and the surrounding environment. Some speech processing techniques such as the hidden Markov models are not suitable for these applications because of the lack of alphabet sounds.

The proposed work is divided into two stages: fault detection, and fault location. The terms location and localization are used interchangeably in this paper. In the fault 
detection stage, the chain codes of the pseudospectrum of the sound signal are used as feature vectors. The extracted chain codes are used as input to dynamic time warping (DTW) techniques for classification. If a sound sample of a motorcycle is identified as faulty in the first stage, it is subjected to the fault location stage. The work employs Mel-frequency cepstral coefficient (MFCC) features for fault location, which are used for speech and speaker recognition. The need, applications, market scenario, and accident-prone Indian rural road conditions motivated this research. The work intends to alert riders to the possibility of accidents by indicating faults well in advance. It is also useful for service station experts in their preliminary analysis of faulty vehicles. The conducted literature survey is divided into two parts: engine fault diagnosis and gearbox fault diagnosis. 1D central contour moments and invariant contour moments of approximation coefficients of DWT have been used as inputs for the DTW classifier for detecting the condition of motorized two-wheelers based on acoustic signals (Anami, Pagi, \& Magi, 2011; Tan \& Wong, 2012). The entropy of db4 wavelet for feature extraction and a functional link neural network has been employed for fault diagnosis of the motorbike engine (Paulraj, Yaacob, \& Mohd Zubir, 2009). A fault diagnosis system has been proposed for a scooter engine platform (Wu, Chang, Liao, Kuo, \& Huang, 2009). The system uses continuous wavelet transform and artificial neural network techniques. The mechanisms of engine front noise generation and the corresponding countermeasures employed in the development of a diesel engine have been surveyed using a sound intensity method (JunHong \& Bing, 2005). A de-noising method uses the Morlet wavelet for extracting the features of sound of vehicle engines with different types of failure.

Localized gear tooth defect recognition by Engin and Gulez (1999) used a wavelet-transform-based artificial neural network. An adaptive wavelet filter based on the Morlet wavelet has been presented for the detection of vibration signals of a gearbox (Lin \& Zuo, 2003). RMS and power spectral density have been used for the fault diagnosis of a Massey Ferguson gearbox (Heidarbeigi, Ahmadi, Omid, \& Tabatabaeefar, 2009). A methodology for gearbox fault location detection used acoustic emission sensors for a split-torque gearbox and used wavelet transform techniques (He, Li, \& Bechhoefer, 2010).

Some of the limitations of the reported works include:

i) Computationally expensive methods and lack of robustness.

ii) Lack of analysis for separability of the features.

iii) Morlet-wavelet-based works are not fast because they have drawbacks such as an absence of a scaling function and not being orthogonal.

iv) Dependency on knowledgebase poses a serious limitation when tested with new data.

v) Lack of real-time applicability.

vi) Lack of transparency due to larger feature sets.

From the comparative analysis of the literature, it is evident that a reasonable amount of research has been reported for a wide range of applications, covering fault classification, vehicle classification, and medical diagnosis. The reported works employ computationally expensive techniques that are not transferable to real-world environments and real-time applications. Comparison of our work with the reported works is difficult because of the differences in experimental conditions, recording 
conditions, and databases. As there is no reported work on fault detection and location in motorcycles, based on their sound patterns, we have taken up this work.

\section{METHODOLOGY}

The proposed methodology has two stages: fault detection and fault location. Fault detection determines whether the motorcycle is healthy or faulty, whereas the fault location stage identifies the exact source of the fault. Figure 1 depicts a block diagram of the proposed methodology.

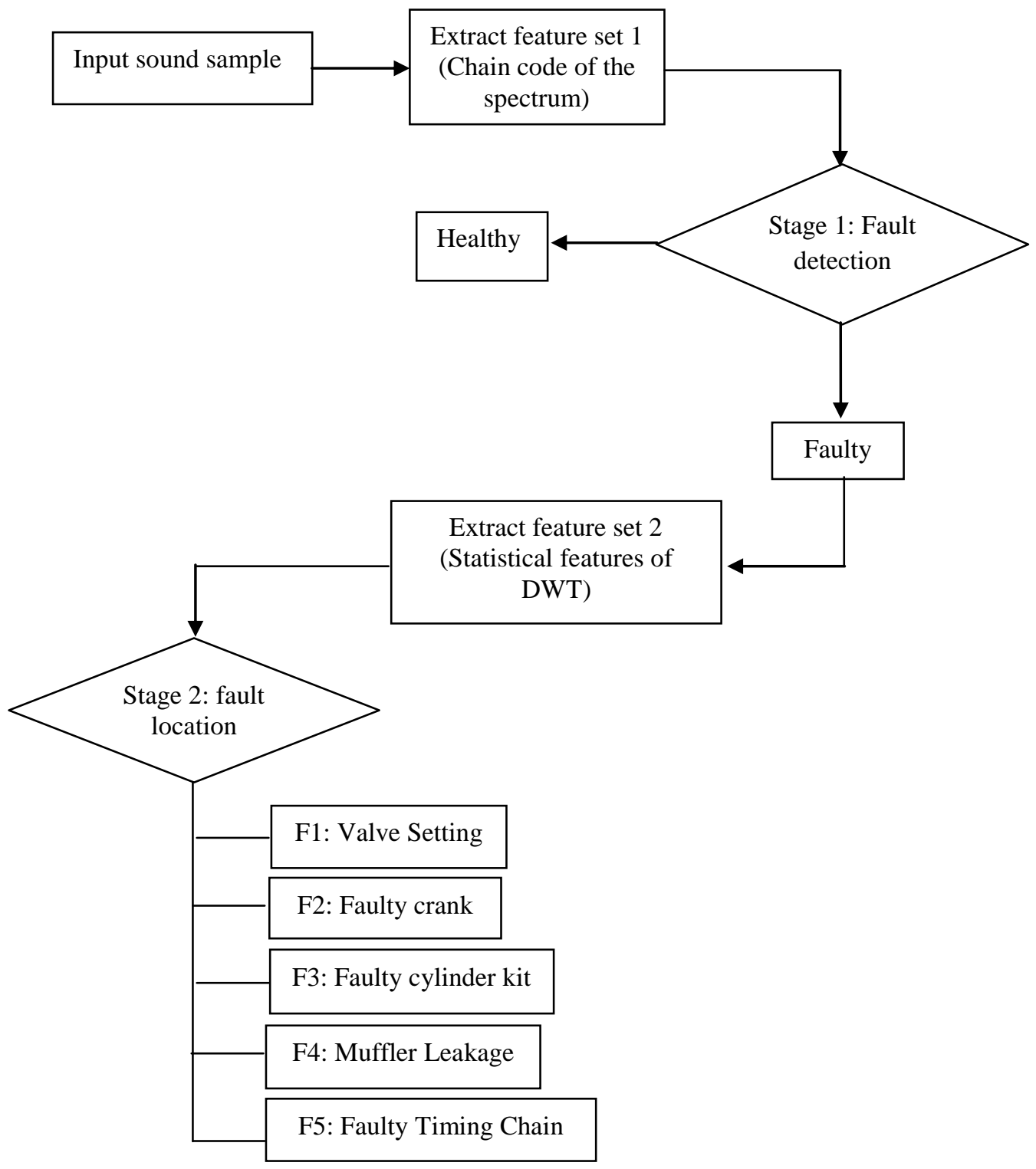

Figure 1. Block diagram of the proposed methodology.

The chain code of the estimated pseudospectrum of length 128 digits is employed as a feature vector. The extracted feature vector is used as input to the DTW classifier. The second stage employs MFCC features of the acoustic signals as input to the DTW classifier. The DTW classifier compares the test feature vector with the reference feature vectors. The smallest of the DTW distances indicates whether the sample is 
healthy or faulty in the first stage, and it indicates the type of fault in the second stage. The reference feature vector for healthy motorcycles is computed as the mode of the chain codes of pseudospectra estimated from the healthy samples. The same procedure is followed for the computation of the reference feature vector for faulty motorcycles.

\section{Stage 1: Fault detection based on chain codes of estimated pseudospectra and DTW}

Fault detection uses the chain code of the estimated pseudospectra as features computed over the recorded sound samples. The following subsections discuss the acquisition of sound samples, and feature extraction and classification, as applied to the fault detection stage.

\section{Acquisition of Sound samples}

A Sony ICD-PX720 digital voice recorder is used for recording the sound samples of motorcycles under idling conditions with no acceleration applied. The recording is performed with $44.1-\mathrm{kHz}$ sampling frequency and 16-bit quantization. The service stations in which the recordings are obtained have additional disturbances from human speech, sounds of other vehicles being serviced, air-compressors, and other automobile repair tools. The recorder is held $500 \mathrm{~mm}$ from the end of the exhaust at the angle of $45^{\circ}$ measured from the exhaust's centerline. The running of the engine and control of throttle are conducted by an expert mechanic. As the recording of samples is conducted in a real-world environment with surrounding disturbances, the method adopted is considered robust to extraneous noise.

Sound samples from healthy motorcycles are collected from those machines that are one-year old, have not passed $6000 \mathrm{~km}$, and that have been serviced regularly. Sound samples of faulty motorcycles are collected from machines of varying age from one- to six-years old and that contain one of the aforementioned faults. 390 sound samples of healthy motorcycles and 390 sound samples of faulty motorcycles are used for the first stage, i.e., fault detection. Table 1 presents the details of the motorcycle sound samples considered in this work. The sound samples of motorcycles from the same manufacturer but from different models are considered for the second stage. A brief description of the faults considered in this work is given below:

Valve setting: Any deviation of between $5-10^{\circ}$ in valve opening/closing causes a considerable rise in peak combustion chamber pressure, which leads to a change in engine sound.

Crank fault: This may occur owing to wear and tear of either oil ring, first ring or second ring.

Muffler Leakage: The reactive gases in the residual exhaust mixed with water vapor create an ideal environment for corrosion reactions. This results in minute holes in the muffler and changes the firing sound of the engine.

Silencer leakage: Silencer leakage is caused either by a hole inside the silencer filter pipe or by a damaged gasket.

Timing Chain: The main function of the timing chain is to operate the valves. A loose chain vibrates and results in a change of sound. 
Table 1. Details of the motorcycles considered.

\begin{tabular}{rlrrlrr}
\hline Sl. No & Model & $\begin{array}{r}\text { Kilomet } \\
\text { ers run }\end{array}$ & Age & Condition & $\begin{array}{r}\text { Recording } \\
\text { duration }\end{array}$ & $\begin{array}{r}\text { No. of } \\
\text { samples }\end{array}$ \\
\hline 1. & HH Spl + & 529 & $2 \mathrm{M}$ & Healthy & $48 \mathrm{~s}$ & 35 \\
2. & HH Spl Pro & 2142 & $4 \mathrm{M}$ & Healthy & $44 \mathrm{~s}$ & 25 \\
3. & HH Pleasure & 676 & $1 \mathrm{M}$ & Healthy & $52 \mathrm{~s}$ & 38 \\
4. & Scoot Streak & 4332 & $11 \mathrm{M}$ & Healthy & $47 \mathrm{~s}$ & 36 \\
5. & Scoot Streak & 1668 & $9 \mathrm{M}$ & Healthy & $46 \mathrm{~s}$ & 37 \\
6. & Scoot Pep + & 756 & $1 \mathrm{M}$ & Healthy & $44 \mathrm{~s}$ & 29 \\
7. & HH spl Pro & 892 & $10 \mathrm{M}$ & Healthy & $51 \mathrm{~s}$ & 41 \\
8. & HH CBZ Ex & 5727 & $1 \mathrm{Y}$ & Healthy & $46 \mathrm{~s}$ & 28 \\
9. & Bajaj CT 100 & 5116 & $1 \mathrm{Y}$ & Healthy & $51 \mathrm{~s}$ & 41 \\
10. & HH Glamour & 1261 & $7 \mathrm{M}$ & Healthy & $62 \mathrm{~s}$ & 42 \\
11. & Pulser DTSI & 5010 & $10 \mathrm{M}$ & Healthy & $49 \mathrm{~s}$ & 38 \\
12. & HH CD100 D & 62979 & $9 \mathrm{Y}$ & Muffler leak & $54 \mathrm{~s}$ & 22 \\
13. & HH Super Spl & 54777 & $5 \mathrm{Y}$ & Muffler leak & $47 \mathrm{~s}$ & 15 \\
14. & HH Passion & 74737 & $6 \mathrm{Y}$ & Silencer leak & $49 \mathrm{~s}$ & 20 \\
15. & HH Spl & 67065 & $10 \mathrm{Y}$ & Silencer leak & $55 \mathrm{~s}$ & 19 \\
16. & HH CD 100 & 39200 & $18 \mathrm{Y}$ & Silencer leak & $54 \mathrm{~s}$ & 16 \\
17. & HH Passion + & 61,999 & $9.5 \mathrm{Y}$ & Timing chain & $50 \mathrm{~s}$ & 38 \\
18. & HH Spl + & 21373 & $4 \mathrm{Y}$ & Timing Chain & $56 \mathrm{~s}$ & 30 \\
19. & HH Spl & 77905 & $6 \mathrm{Y}$ & Timing Chain & $50 \mathrm{~s}$ & 31 \\
20. & HH CD Dawn & 73888 & $6 \mathrm{Y}$ & Timing chain & $48 \mathrm{~s}$ & 30 \\
21. & HH Pleasure & 27043 & $4 \mathrm{Y}$ & Timing Chain & $49 \mathrm{~s}$ & 32 \\
22. & HH CBZ & 81014 & $8 \mathrm{Y}$ & Timing chain & $64 \mathrm{~s}$ & 49 \\
23. & HH Spl + & 15711 & $2 \mathrm{Y}$ & Valve setting & $51 \mathrm{~s}$ & 13 \\
24. & HH Splend & 50632 & $5 \mathrm{Y}$ & Valve setting & $58 \mathrm{~s}$ & 22 \\
25. & HH Splend & 36040 & $3 \mathrm{Y}$ & Valve setting & $46 \mathrm{~s}$ & 17 \\
26. & HH Spl & 4,216 & $8 \mathrm{M}$ & Faulty crank & $47 \mathrm{~s}$ & 36 \\
& & & & Total No. of samples & 780 \\
\hline & & & & &
\end{tabular}

\section{Segmentation}

The acquired sound samples are segmented into samples, each of one-second duration, for uniformity in processing. The first segment is extracted beginning from the local maxima in the first 50-ms span. The subsequent segment begins at the local maxima of the next 50-ms duration from the end of the previous segment.

\section{Feature Extraction}

The slopes of the pseudospectral spans are employed as features. The estimated pseudospectral vector is divided into eight equal spans. The slope of each segment is computed to form a feature vector. 


\section{Pseudospectrum Estimation}

The pmusic function of MATLAB is employed for pseudospectral estimation of the sound signals. The pseudospectrum is calculated by using estimates of the eigenvectors of a correlation matrix associated with the input data $x$. $[\mathrm{S}, \mathrm{w}]=\operatorname{pmusic}(x, p)$ implements the MUSIC (Multiple Signal Classification) algorithm and returns $S$, the pseudospectrum estimate of the input signal $x . p$ is the signal subspace dimension and $w$ is a vector of normalized frequencies (in rad/sample) at which the pseudospectrum is evaluated. The MUSIC estimate is given by Eq. (1).

$$
P_{\text {music }}(f)=\frac{1}{e^{H}(f)\left(\sum_{k=p+1}^{N} v_{k} v_{k}^{H}\right) e(f)}=\frac{1}{\sum_{k=p+1}^{N}\left|v_{k}^{H} e(f)\right|^{2}}
$$

where $N$ is the dimension of the eigenvectors and $v_{k}$ is the $k^{\text {th }}$ eigenvector of the correlation matrix of the input signal. The integer $p$ is the dimension of the signal subspace, such that the eigenvectors $v_{k}$ used in the sum correspond to the smallest eigenvalues and also span the noise subspace. The vector $e(f)$ consists of complex exponentials, such that the inner product $v_{k}^{H} e(f)$ amounts to a Fourier transform. Eq. (2) shows how the summation is weighted by the eigenvalues of the correlation matrix, as in the eigenvector method.

$$
P_{e v}(f)=\frac{1}{\left(\sum_{k=p+1}^{N}\left|v_{k}^{H} e(f)\right|^{2}\right) / \lambda_{k}}
$$

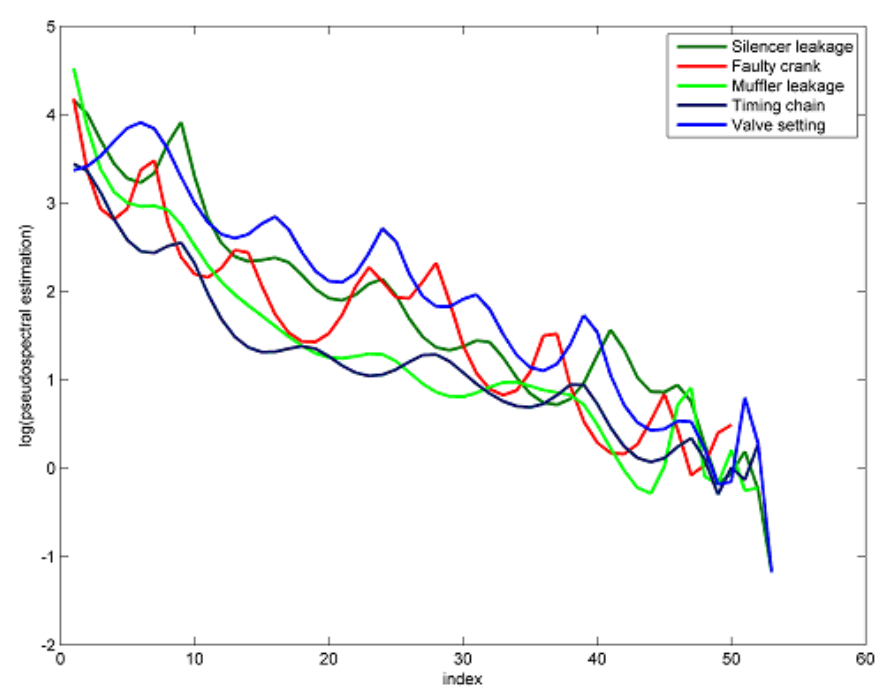

Figure 2. Logarithmic plot of pseudospectral estimates of faults.

The function relies on the singular value decomposition (SVD) matrix decomposition in the signal case, and it uses the eig function for analyzing the correlation matrix. Logarithmic plots are used to analyze the separability exhibited by the pseudospectra for different faults considered in this study. Figure 2 shows the 
logarithmic plot of the pseudospectral estimates for different faults. The spectral estimates differ for each fault, resulting in easier fault localization. From the logarithmic plot of the pseudospectra, it can be observed that the fault signatures for different faults differ in various frequency bands. This can be attributed to the variations in engine cycles and exhaust properties, which result in the changes of sound under different fault conditions. The fault signatures of motorcycles are non-stationary in nature and thus, wavelet-based techniques are more suitable for the analysis of such non-stationary signals. Furthermore, there is scope for the analysis of the severity of the fault based on the sound and its characteristics.

\section{Chain Code of a Spectral Segment}

The pseudospectrum of a sound signal computed with pmusic(x, 16) has 129 elements. The chain code of a spectral segment is generated based on the changes in the gradient directions of the pseudospectrum from left to right. The directions in which the spectral gradient changes are 0 (right), 1 (top-right), and 7 (bottom-right). As adjacent values in the estimated pseudospectrum vector are considered for computing the chain code, the generated chain code has 128 values. The trailing 0s of the chain code vectors are removed to yield the reduced chain codes of non-uniform lengths. Hence, the DTW algorithm is used for comparing the reduced chain codes of unequal lengths. The chain code vector of a spectral curve is computed as:

$$
\text { ChCode }(i)= \begin{cases}0 & x(i)=x(i+1) \\ 7 & x(i)>x(i+1) \\ 1 & x(i)<x(i+1)\end{cases}
$$

where $1 \leq i \leq 128$.

The reference feature vector for healthy motorcycle samples is constructed by taking the mode of the respective chain codes. Similarly, the reference feature vector for faulty motorcycles is constructed. The test feature vectors are compared against the reference feature vectors of healthy and faulty motorcycles.

\section{Computation of Mode of Chain Code Vectors}

The mode of a list is the element with the highest frequency of occurrence. The mode of each column of a set of feature vectors is computed. The reference feature vector is generated by concatenating the mode of the first column followed by the mode of the second column and so on. The trailing zeros of the chain codes are removed resulting in vectors of varying lengths. DTW is adjudged suitable for classification, because it can compare two vectors of unequal lengths. The reference feature vector for healthy motorcycle samples in terms of reduced chain code is:

$$
\begin{array}{r}
7,7,7,7,1,1,1,1,1,1,1,1,1,1,7,7,7,7,7,7,1,1,1,1,1,1,1,1,1,1,1,1,1,1,1,1,1,1,1,1,1,1,1,1,1,1 \\
, 1,1,1,1,1,1,1,1,1,1,1,1,1,1,1,1,1,0,1,0,1,0,0,1,0,0,0,0,0,0,0,0,0,0,0,0,0,7,0,0,0,0,0,0,0,0,0 \\
, 0,0,0,1,0,0,0,0,0,0,0,1,0,0,0,0,0,0,0,0,0,0,0,0,7
\end{array}
$$

In a similar fashion, the reference faulty feature vector is computed as the mode of the feature vectors of faulty samples, which is given as: 
$7,7,1,1,1,1,1,1,1,1,1,1,1,1,1,1,1,1,1,1,1,1,1,1,1,1,1,7,7,7,7,7,7,1,1,1,1,1,1,1,1,1,1,1,1,1$ $, 1,1,1,1,1,1,1,1,1,1,1,1,1,1,1,1,1,1,1,1,0,1,1,0,1,0,1,0,0,0,1,0,0,0,0,0,0,0,0,0,0,0,0,0,0,0,0$ $, 0,0,0,0,0,0,0,0,0,0,0,0,0,0,0,0,0,1,0,0,7$

\section{DTW Classifier}

The DTW algorithm (Sakoe and Chiba, 1978) is used to calculate an optimal warping path between two time series and the distance between them. It matches those patterns independent of non-linear variations. Consider the two numerical sequences $\left(a_{1}, a_{2}, \ldots, a_{n}\right)$ and $\left(b_{1}, b_{2}, \cdots, b_{m}\right)$. The local distances between the elements of the two sequences are calculated, which results in a matrix of distances of size $(n \times m)$ :

$$
d_{i j}=\left|a_{i}-b_{j}\right|, \quad i=1, \ldots, n \quad j=1, \ldots, m
$$

The local distance matrix is used to compute the minimal distance matrix between the two sequences, using a dynamic programming approach and the optimization criterion, as given in Eq. (5):

$$
a_{i j}=d_{i j}+\min \left(a_{i-1, j-1}, a_{i-1, j}, a_{i, j-1}\right)
$$

where $a_{i j}$ is the minimal distance between the subsequences $\left(a_{1}, a_{2}, \ldots, a_{n}\right)$ and $\left(b_{1}, b_{2}, \cdots, b_{m}\right)$. A warping path is obtained through the minimal distance matrix from $a_{11}$ element to element $a_{n m}$, consisting of those $a_{i j}$ elements that have formed the $a_{n m}$ distance. The global warp cost of the two sequences is defined as in Eq. (6):

$$
G C=\frac{1}{p} \sum_{i=1}^{p} w_{i}
$$

where $w_{i}$ are those elements that belong to the warping path and $p$ is the number of elements on the warping path.

The DTW distance of the test feature vector against the reference feature vector is computed. The algorithm finds an optimal match by warping the sequences to determine a measure of their similarity. DTW matches the patterns independent of non-linear variations. If the Euclidian distance of the test sample with the healthy mean DTW distance is smaller than that with the faulty mean DTW distance, the motorcycle is classified as healthy; otherwise, it is classified as faulty. Figure 3 illustrates the working of the DTW algorithm. It compares the feature vectors $(0.5,0.9,1.2,0.8,0.6,0.5,0.2$, $0.1)$ and $(0.7,0.6,1.1,0.7,0.6,0.4,0.3,0.2)$. The smallest of the distances of the test feature vector to the reference feature vectors of the healthy and faulty samples, indicates the health condition in the first stage. The same criterion is used to indicate the type of the fault in the second stage. 


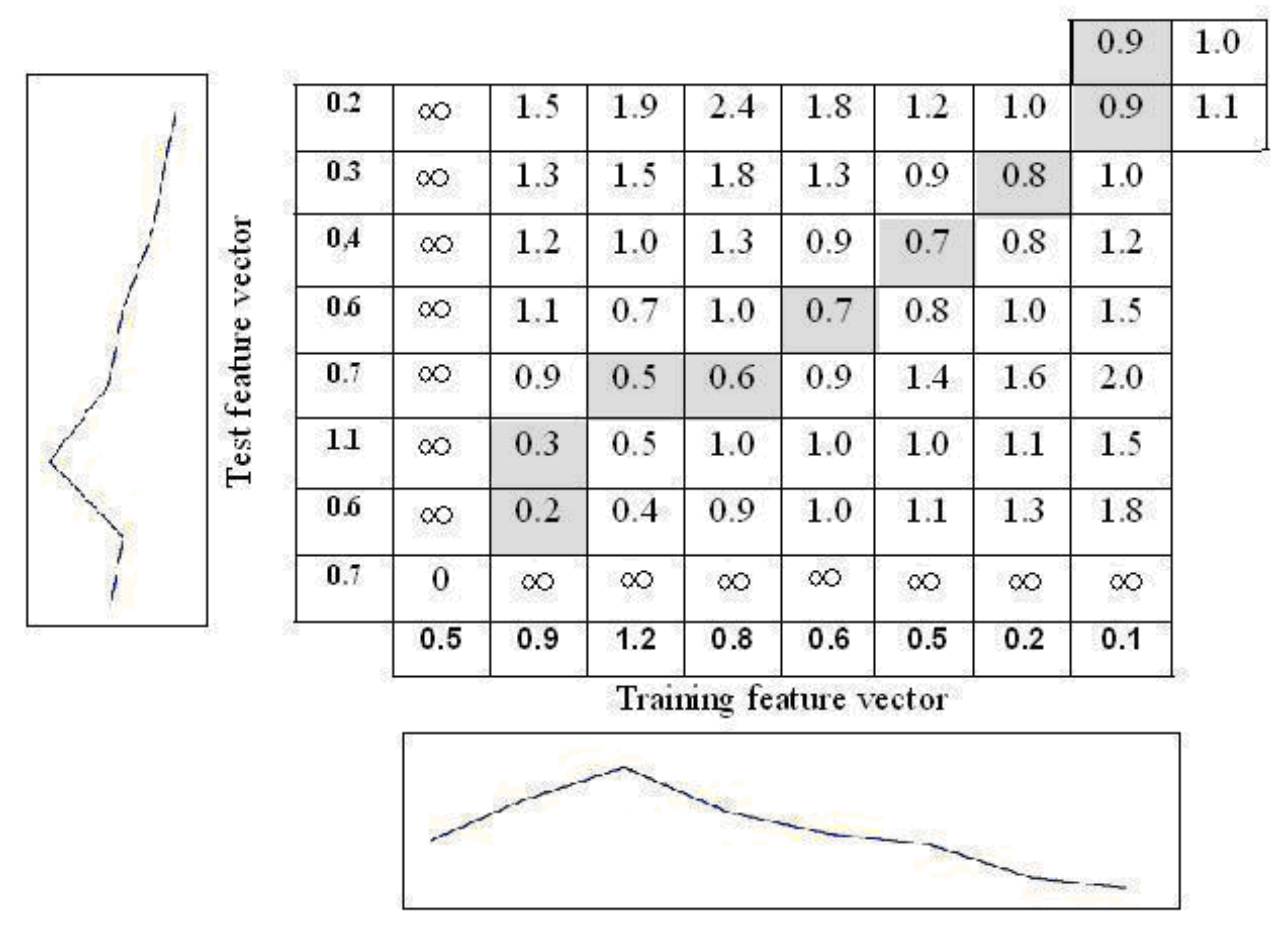

Figure 3. Illustration of working of DTW algorithm.

\section{Stage 2: Fault Location using MFCC Features}

MFCCs are derived from a cepstral representation of the sound signal. The Mel-scale approximates the human auditory system's response more closely than the linearly spaced frequency bands used in the normal cepstrum. The frequency bands are equally spaced on the Mel-scale. The MFCCs are computed first by taking the Fourier transform of a signal. Then, the powers of the spectrum are mapped onto the Mel scale using triangular overlapping windows. The logs of the powers at each of the Mel-frequencies are taken. A discrete cosine transform of the list of Mel log powers is performed. Finally, the amplitudes of the resulting spectrum, taken as MFCCs, are used as features in speech recognition, speaker recognition systems, and music information retrieval applications, such as genre classification, audio similarity measures, etc. MFCC values are noise sensitive for additive noise and hence, are not robust. MFCC features may not be optimal for vehicle fault diagnosis because the filter bank spreads out the fine details of the spectral patterns. Sometimes these details contribute significantly to the success of classification.

The motorcycle sound samples classified as faulty in the first stage are used as input for the fault location stage. The eight MFCCs are computed on a signal of onesecond duration and used as features for fault localization. The reference feature vectors for this stage are computed as the mean of the feature vectors of respective fault types. The DTW algorithm compares the test feature vectors with the reference features of the five fault types. 


\section{RESULTS AND DISCUSSION}

Disjoint sample sets are used for training and testing. The sample set includes 390 samples of healthy motorcycles and 390 samples of faulty motorcycles. If $n$ is the number of samples used for training, then $(390-n)$ is the number of samples used for testing. For the first stage, the samples from healthy motorcycles of different brands and faulty motorcycles of the Hero Honda brand are considered. The following criterion is used for classification.

$$
\begin{aligned}
& \text { If }((\text { EucDist }(\text { Test, meanDTW }(\mathrm{H})<\text { EucDist(Test, meanDTW(F) }) \\
& \text { Class = Healthy; } \\
& \text { else } \\
& \quad \text { Class = Faulty; }
\end{aligned}
$$

where EucDist $(\mathrm{x}, \mathrm{y})$ - Euclidean distance of the vector $\mathrm{x}$ with vector $\mathrm{y}$, Test - Test vector, meanDTW $(\mathrm{H})$ - Mean of the DTW distances of healthy samples, i.e., reference vector for healthy samples;

meanDTW(F) - Mean of the DTW distances of faulty samples, i.e., reference vector for faulty samples;

Class - Health condition of the motorcycle.

The results of the first stage are shown in Figure 4.

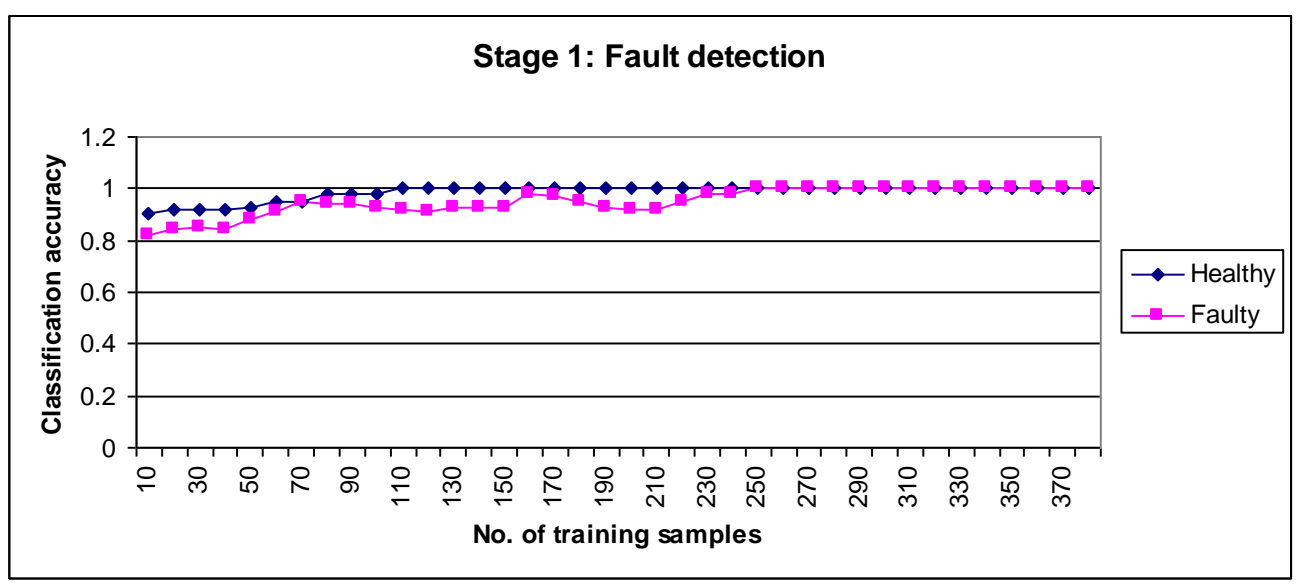

Figure 4. Performance of the classifier for fault detection.

Table 2 shows the confusion matrix for the fault location stage. Table 3 summarizes the classification performances for different fault types, which shows that the classification accuracy is 0.8108 for fault type 3. Classification accuracy of 1.0000 is attained in the case of fault type 2. Out of 390 samples considered, 369 samples are correctly classified, yielding the overall classification accuracy of 0.9453 . From Table 3 , it can be inferred that the MFCC features, which are employed for speech recognition, can be applied for non-speech applications. The signals are not preprocessed for denoising, but the results are quite satisfactory and hence, the features and the methodology are found to be robust. DTW is suitable for classifying samples with a limited database. Use of dynamic programming ensures a polynomial complexity 
of $O\left(n^{2} v\right)$, where $n$ is length of the sequence and $v$ is the number of samples in the training set. However, the $O\left(n^{2} v\right)$ complexity is not satisfactory for a large database. DTW is appropriate for applications that need simple word recognition, reliability tests in manufacturing units, initial servicing activities in service stations, speech recognition in telephones, etc.

Table 2. Confusion matrix for fault location.

\begin{tabular}{|c|c|c|c|c|c|}
\hline & F1 & F2 & F3 & F4 & F5 \\
\hline F1 & 49 & 0 & 0 & 0 & 3 \\
\hline F2 & 0 & 36 & 0 & 0 & 0 \\
\hline F3 & 0 & 0 & 30 & 0 & 7 \\
\hline F4 & 0 & 0 & 0 & 54 & 0 \\
\hline F5 & 1 & 7 & 0 & 0 & 210 \\
\hline
\end{tabular}

Table 3. Classification performance for different faults.

\begin{tabular}{|c|c|c|c|c|c|c|}
\hline $\begin{array}{l}\text { Fault } \\
\text { type }\end{array}$ & Fault & $\begin{array}{c}\text { Total No. of } \\
\text { samples }\end{array}$ & $\begin{array}{c}\text { No. of samples } \\
\text { correctly } \\
\text { classified }\end{array}$ & $\begin{array}{c}\text { No. of } \\
\text { samples } \\
\text { misclassified }\end{array}$ & $\begin{array}{c}\text { No. of } \\
\text { samples } \\
\text { not } \\
\text { classified }\end{array}$ & $\begin{array}{c}\text { Classification } \\
\text { accuracy }\end{array}$ \\
\hline F1 & Valve setting & 52 & 49 & 3 & 0 & 0.9455 \\
\hline $\mathrm{F} 2$ & Faulty crank & 36 & 36 & 0 & 0 & 1.0000 \\
\hline F3 & $\begin{array}{l}\text { Muffler } \\
\text { leakage }\end{array}$ & 37 & 30 & 7 & 0 & 0.8108 \\
\hline F4 & $\begin{array}{l}\text { Silencer } \\
\text { leakage }\end{array}$ & 55 & 54 & 0 & 1 & 0.9818 \\
\hline \multirow[t]{2}{*}{ F5 } & Timing chain & 210 & 200 & 8 & 2 & 0.9524 \\
\hline & Total & 390 & 369 & 18 & 3 & 0.9461 \\
\hline
\end{tabular}

\section{CONCLUSION}

A two-stage methodology for the fault diagnosis of motorcycles, based on sound patterns, is presented in this paper. The fault detection stage uses the chain codes of the estimated pseudospectra of the sound samples. Fault location is attempted by using MFCC features of the sound samples. Both stages employ the DTW classifier. The results for fault location differ for each type of fault. This can be attributed to the similarity of the sounds of the faults belonging to the same subsystem. The overall classification rate for fault location is around 94\%. This work leaves future scope for fault location in different subsystems of motorcycles and for the detection of multiple faults. It finds applications in acoustic-signal-based machine fault detection and location, cardiac signal analysis, musical instrument tuning, and spoken-word analysis, etc. 


\section{ACKNOWLEDGEMENTS}

We the authors thank Prof. Ramesh Wadawadagi and Prof. Vishwanath Kagawade for language corrections.

\section{REFERENCES}

Anami, B. S., Pagi, V. B., \& Magi, S. M. (2011). Wavelet based acoustic analysis for determining health condition of motorized two-wheelers. Journal of Applied Acoustics, 72(7), 464-469.

Engin, S. N., \& Gulez, K. A. (1999). Wavelet transform-artificial neural networks (WTANN) based rotating machinery fault diagnostics methodology. IEEE NSIP' 99, Falez Hotel, Antalya, Turkey, pp. 714-720.

He, D., Li, R., \& Bechhoefer, E. (2010). Split torque type gearbox fault detection using acoustic emission and vibration sensors. IEEE International Conference on Networking, Sensing and Control (ICNSC), pp. 62-66.

Heidarbeigi, K., Ahmadi, H., Omid, M., \& Tabatabaeefar, A. (2009). Fault diagnosis of Massey Ferguson gearbox using power spectral density. Journal of Agricultural Technology, 5(1), 1-6.

IR (India-Reports on February 20). (2010). Retrieved from http://india-reports.in/futuregrowth-global-transitions/economy-in-transition/two-wheeler-segment-in-india/ (Accessed on 12-09-2010).

JunHong, Z., \& Bing, H. (2005). Analysis of engine front noise using sound intensity techniques. Journal of Mechanical Systems and Signal Processing, 19, 213-221.

Lin, J., \& Zuo, M. J. (2003). Gearbox fault diagnosis using adaptive wavelet filter, Journal of Mechanical Systems and Signal Processing, 17(6), 1259-1269.

MFCC (Mel-Frequency Cepstral Coefficients). (2012). Retrieved from http://en.wikipedia.org/wiki/Mel-frequency_cepstrum.

Paulraj, M. P, Yaacob, S., \& Mohd Zubir, M. Z. (2009). Motorbike engine faults diagnosing system using entropy and functional link neural network in wavelet domain. Proc. of the Int. Conf. on Man-Machine Systems (ICoMMS) 11-13 October 2009, Batu Ferringhi, Penang, MALAYSIA, pp. 2B4-1-2B4-5.

PMUSIC function of MATLAB, http://www.mathworks.in/help/toolbox/signal/ pmusic.html, (Accessed on 10-11-2012).

Sakoe, H., \& Chiba, S. (1978). Dynamic programming algorithm optimization for spoken word recognition. IEEE Transactions on Acoustics, Speech and Signal Processing, 26(1): 43-49.

Tan, C. L., \& Wong, S. V. (2012). Motorcycle crash test center: a movable barrier approach. International Journal of Automotive and Mechanical Engineering, 5, 630-638.

Wu, J. D., Chang, E. C., Liao, S. Y., Kuo, J. M., \& Huang, C. K. (2009). Fault classification of a scooter engine platform using wavelet transform and artificial neural network. Proc. of the International Multi Conference of Engineers and Computer Scientists, IMECS 2009, March 18-20, 2009, Hong Kong, 1, 58-63. 